\title{
Emergence of Conduction Channels in Lithium Silicate
}

\author{
Heiko Lammert* and Andreas Heuel| \\ Institute of Physical Chemistry and Sonderforschungsbereich 458, Corrensstraße 30, D-48149 Münster, Germany
}

(Dated: March 14, 2021)

\begin{abstract}
The existence of conduction channels in lithium silicate $\left(\mathrm{Li}_{2} \mathrm{O}\right)\left(\mathrm{SiO}_{2}\right)$ is investigated. Regions of the system where many different ions pass by form channels and are thus spatially correlated. For a closer analysis the properties of the individual ionic sites are elucidated. The mobility of ions in single sites is found to depend strongly on the number of bridging oxygens in the coordination shell. The channels are not reflected in the network structure as obtained from the distribution of the bridging oxygens. Spatial correlations similar to those found in the silicate also emerge from studying the dynamics of particles in a simple random lattice model. This supports the suggestion that the observed spatial correlations can be viewed in analogy to the emergence of percolation paths.
\end{abstract}

\section{INTRODUCTION}

The presence of conduction pathways has been proposed to rationalize the fast conduction in ion conductors like alkali silicates. Based on EXAFS data on the coordination environments in these systems, Greaves proposed the modified random network model for glasses ${ }^{1}$. It predicts the aggregation of modifier ions into channels lined by non-bridging oxygen atoms. Later NMR studies 2.3 reported both inhomogeneous and homogeneous distributions of cations in the structure with a clustering of alkali ions in the low concentration regime. These results are backed by molecular dynamics (MD) simulations of alkali silicate where also only for low alkali concentrations a mild clustering of alkali ions has been found ${ }^{4,5}$.

In his analysis of MD simulations of sodium silicate, Jund et $\mathrm{a}^{1}{ }^{6}$ focussed on the most mobile cations. They introduced $n_{\text {diff }}$, the number of different ions visiting a given subvolume, to determine those parts of the system taking part in effective long range transport. They report that the regions of the silicate network, visited by the largest number of different ions form a network of blobs and connecting channels (denoted conduction channels). Stated differently, these regions show a significant spatial correlation beyond the nearest-neighbor shell. In particular, these spatial correlations are much stronger than any possible correlation of alkali positions themselves. Thus these conduction channels do not simply emerge from clustering of alkalis 6 .

Interestingly, all static structure factors for sodiumsilicate exhibit a prepeak at $q_{1} \approx 0.95 \AA^{-1}$, corresponding to the next-nearest $\mathrm{Na}-\mathrm{Na}$ or $\mathrm{Si}-\mathrm{Na}$ neighbors, as shown experimentally ${ }^{7}$ and numerically ${ }^{8,9}$. Thus the structure displays some long-range correlations. One may be tempted to relate these long-range correlations to the channels discussed above. The validity of this conclusion, however, is not obvious, since the structure refers to all ions whereas the channels emerge from the properties of the specific subset of regions, visited by many different ions.

In this paper we analyze the question to which degree these conduction channels are predetermined by the structure. This question is central because it elucidates the role of the network for long-range transport. Two extreme scenarios are conceivable. First, following the concepts of Greaves, the network may supply channels where ion conduction is strongly favored due to the aggregation of the relevant structural elements (in Greaves work: non-bridging oxygens) which favor fast dynamics. In this scenario these relevant structural elements would also display long-range spatial correlations. Second, the structure might only determine the local mobility of an ion such that the relevant structural elements do not display these long-range correlations. Rather the formation of conduction channels is a simple statistical process in analogy to the formation of percolation paths in random-energy or random-barrier lattice models of ion conduction 10

In previous work we have presented a method how to identify individual lithium sites which are basically timeinvariant below the glass transition ${ }^{11}$. In particular, we could show that the continuous trajectories of the lithium ions can be interpreted as hops between these sites. With this information at hand we can identify the conduction channels by counting the number of different ions visiting a specific site during a long MD simulation. This discretization of the system is somewhat more adapted to the conduction process than the simple tiling by small cubes as done by Jund et $\mathrm{a}^{16}$. In particular, we are very sensitive to the effect of structural elements like bridging or non-bridging oxygens on the alkali dynamics.

The paper is organized as follows. In Section III we discuss the technical aspects of our work. Section III contains the results which are finally discussed in Section IV

\section{TECHNICAL ASPECTS}

The data for this investigation are based on MD simulations of lithium silicate $\left(\mathrm{Li}_{2} \mathrm{O}\right)\left(\mathrm{SiO}_{2}\right)$. The system containing 1152 atoms was propagated under NVT conditions at $640 \mathrm{~K}$ with 2 fs stepsize for $10 \mathrm{~ns}$ simulation time. As a basis for investigations of the cation dynamics we first located the individual sites available to the lithium ions. For this purpose we have discretized the 
system in very small cubes $(0.3 \AA)^{3}$ and determined the occupation number in every cube. This is a simple way to sample the effective potential energy landscape provided by the silicate network. In order to distinguish regions which indeed belong to ionic sites and those which only serve as transition paths between these sites we have used some reasonable cutoff criterion for the identification of those cubes which are part of ionic sites. Subsequent cluster analysis of the remaining cubes finally defines the ionic sites. For our system we have obained 378 different sites. Details about the method and about the properties of the sites are given in an earlier paper $\stackrel{11}{\underline{11}}$.

We characterize the mobility of a site in two different ways. First, in analogy to the work by Jund et al. the number of different ions visiting a site has been determined. It is denoted $n_{\text {diff }}$. Sites for which the value of $n_{\text {diff }}$ belongs to the $10 \%$ highest are denoted A-sites. In contrast, those with the $10 \%$ smallest values of $n_{\text {diff }}$ are denoted $\bar{A}$-sites. Second, we determined the average residence time in a site, $\tau$. Analoguosly, sites with the $10 \%$ shortest residence times are denoted B-sites, those with the longest $10 \%$ residence times $\bar{B}$-sites. In general terms, A- and B-sites indicate positions in the network where ions are fast, $\bar{A}$ - and $\bar{B}$-sites where ions are slow. In this investigation both quantities will be used together as different measures of site mobility.

Furthermore we performed straightforward Monte Carlo simulations for hopping particles on a 2D square lattice model with 1600 sites and periodic boundary conditions. We introduced random site energy disorder and additional barrier disorder among adjacent sites. Both distributions were chosen as constant with energies between 0 and $8 k_{B} T$. $90 \%$ of all sites were populated and the hopping particles interacted via simple excluded volume interaction. The energy disorder was kept constant during the whole run.

\section{RESULTS}

\section{A. General properties of sites}

The two definitions of site mobility are compared in Fig. 11 which shows $n_{\text {diff }}$ plotted against $\tau$ for all sites. The highest possible value of $n_{\text {diff }}$ for a site is basically given by the number of residences permitted by their average duration $\tau: n_{\text {diff }}<=t / \tau$. This implies that a sufficiently short average residence time is a necessary condition for a large number of different ions visiting a site. A broad range of lower values for $n_{\text {diff }}$ is also found, caused by multiple visits of identical ions. The correlation between $\tau$ and $n_{\text {diff }}$ is therefore only weak.

General information on the structural environment of the sites can be obtained from the radial distribution function $\mathrm{g}(\mathrm{r})$ of network atoms around lithium. It is shown in Fig. 2f for bridging oxygens (BOs), non-bridging oxygens (NBOs), and silicon atoms. BOs and NBOs have been distinguished according to the number of silicon

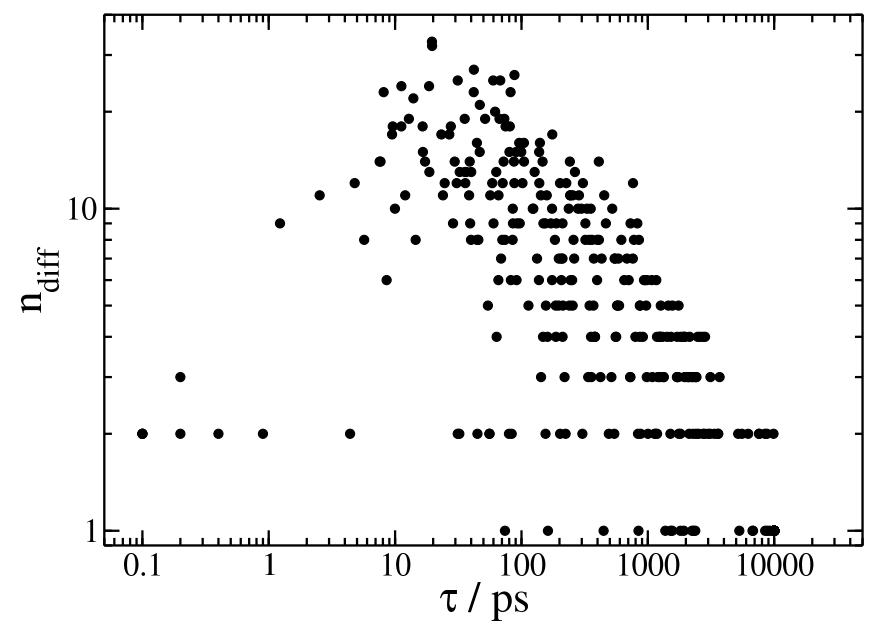

FIG. 1: Scatter plot of the number of different ions $n_{\text {diff }}$, vising a site vs. the average residence time of a site $\tau$.

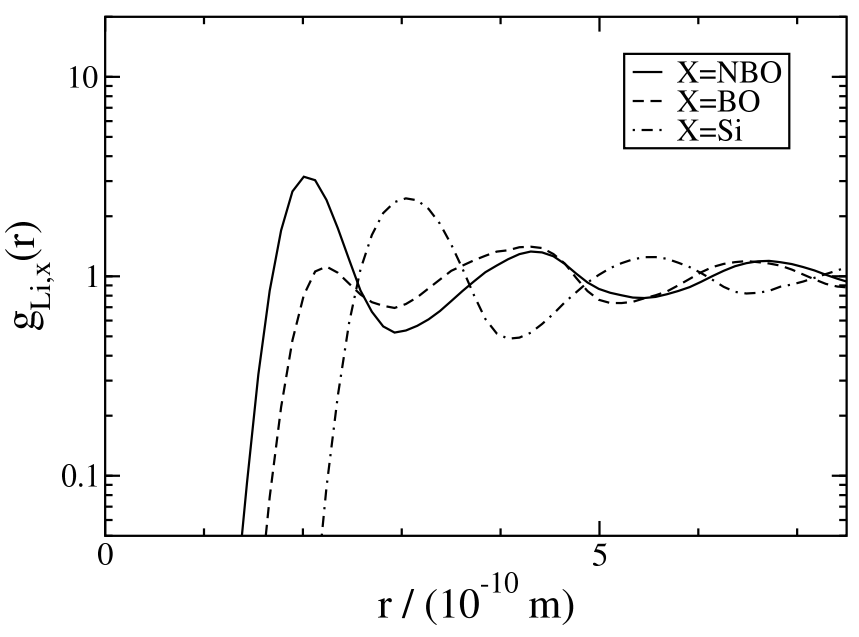

FIG. 2: Mixed $\mathrm{g}(\mathrm{r})$ for $\mathrm{Li}$ and network species

atoms within $2.4 \AA$, the distance of the first minimum in the $\mathrm{g}(\mathrm{r})$ of silicon and oxygen. One finds that lithium atoms are surrounded by oxygens. Integration up to the first minimum gives average coordination numbers of 4.0 NBOs and 1.2 BOs. In contrast, if the average number of 5.2 oxygen neighbors were divided according to the relative numbers of BOs and NBOs in the system one would obtain 3.3 NBOs and $1.8 \mathrm{BOs}$. As expected, NBOs are favored as coordination partners of lithium 1.12.13.14.

\section{B. Spatial correlations of sites}

Now we analyze the question to which degree the mobilities of the different sites are correlated. The presence of channels, defined as regions of the system where many different ions pass would imply that A-sites (and correspondingly $\bar{A}$-sites) are spatially correlated over larger distances. Furthermore, also possible spatial correlations 


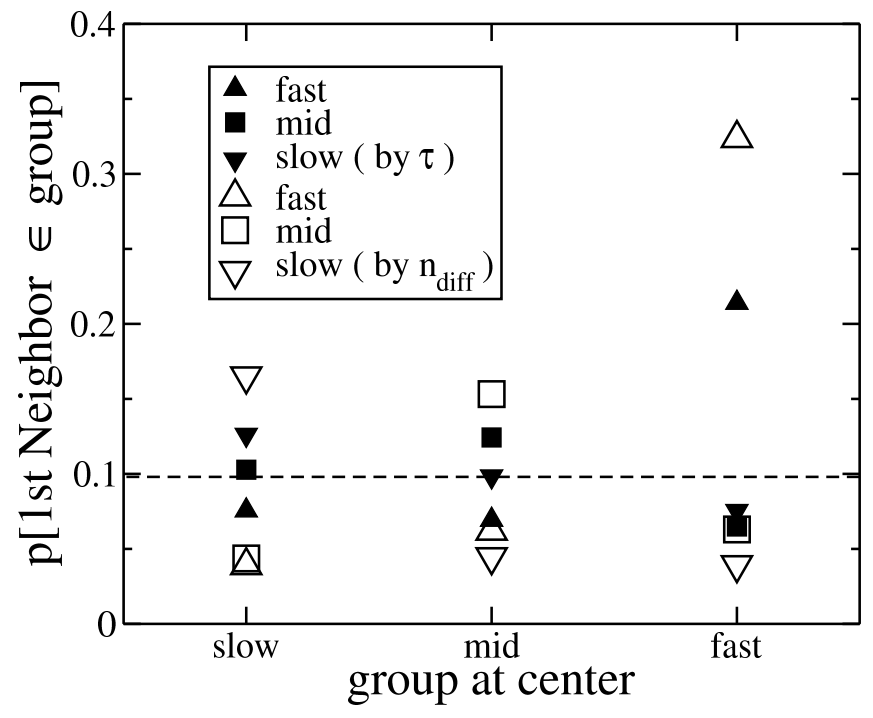

FIG. 3: Correlation of mobilities to the next neighbor shell of sites

of B-sites are analyzed.

It can be expected that correlations on the length scale of the nearest-neighbor distance occur due to trivial reasons. For a fast B-site it can be expected that there is a low barrier to an adjacent site. Accordingly, also the residence time in this adjacent site can be expected to be small because a fast backjump to the original site would be possible. This trivial correlation is limited to next neighbors. If B-sites were arranged into channels, they must show an additional correlation over longer distances. The presence of a continuous pathway of B-sites through the system would result in an increased probability to find other B-sites among the second neighbors of a given B-site.

Apart from A- and $\bar{A}$-sites (and analogously B- and $\bar{B}$-sites) we also selected a subset of $10 \%$ of sites with intermediate $\tau$ and $n_{\text {diff }}$, respectively. Each of the three groups contained 37 sites, i.e. ca. $10 \%$ of the total number. Because of the low number of sites we did not compute their complete pair distribution functions $\mathrm{g}(\mathrm{r})$. Instead we counted directly the number of neighbors from each of the three groups around a given central site. The limits of the first and second neighbor shell were taken from the minima in the $\mathrm{g}(\mathrm{r})$ of all sites at $4.2 \AA$ and $6.8 \AA$. The results for slow, intermediate and fast central sites are compared in Fig. 3 and Fig. 4 for the first and for the second shell respectively.

All values are given as a fraction of the total number of neighbors. The statistical value of $37 / 378=9.8 \%$ for the contribution of each group is shown by the dashed lines. Generally, there is a clear tendency that a site will be surrounded by first neighbors of similar mobility. The strongest correlation is found among the fast sites. While the findings are similar for both $\tau$ and $n_{\text {diff }}$, they are more pronounced in the latter case. For the second shell of neighbors, the correlations almost vanish

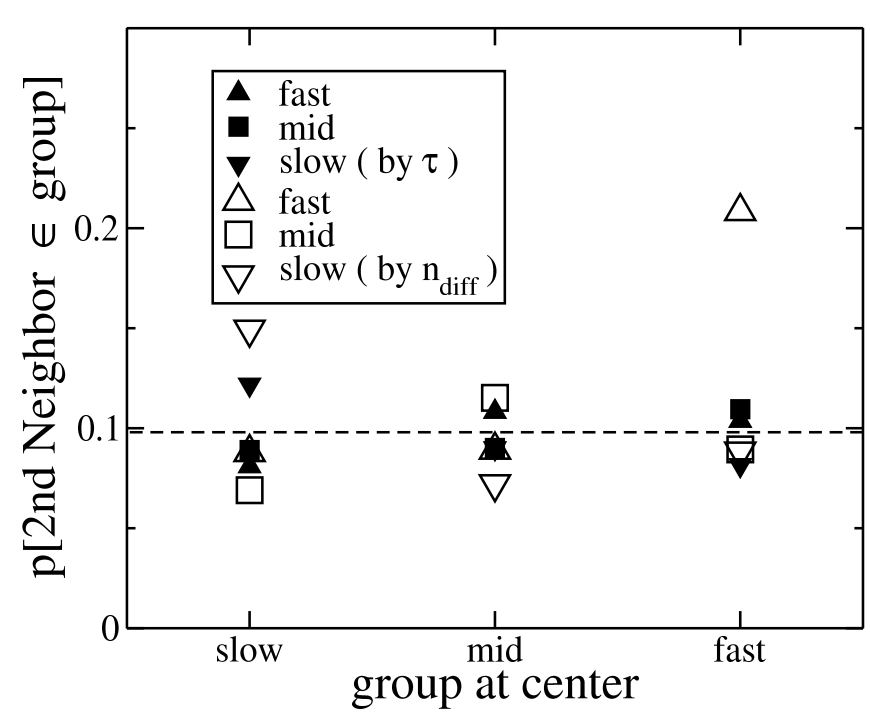

FIG. 4: Correlation of mobilities to the second next neighbor shell of sites

for the residence time $\tau$, i.e. B-sites (and $\bar{B}$-sites) are spatially not correlated. In contrast, as already reported by Jund et al. A-sites are significantly correlated beyond the nearest-neighbor shell. The correlation for $\bar{A}$-sites is much weaker, but still present.

\section{Spatial correlations of relevant network properties}

Next we turn to the question in which sense the conduction channels are predetermined by the structure. To answer this question we have to proceed in two steps. First, we have to check which property of the network significantly favors highly mobile sites. As will be shown below it is the presence of bridging oxygens (BOs) which displays the strongest correlation with the mobility of a nearby site. Second, possible long-range spatial correlations of this network property (here: occurrence of BOs) have to be analyzed. More specifically we check whether sites with a high/low number of BOs are spatially correlated. Again we have to be aware that trivial nearest-neighbor correlations will be present. If some site displays an increased number of BOs as compared to the average site it can be expected that a small number of neighbor sites shows the same correlation because one BO typically belongs to 2 lithium ions.

Using the sets of sites already defined above, we first count the number of BOs, NBOs and $\mathrm{Si}$ atoms up to the 1st minimum distances of $2.9 \AA, 3.0 \AA$, and $4.1 \AA$ respectively. The results are shown in Fig. [5] As expected from the above-mentioned analysis of coordination numbers, sites are on average surrounded by $\approx 4$ NBOs, while only 0.5 to $1.5 \mathrm{BOs}$ are found. For comparison, lines are shown for $1.8 \mathrm{BOs}$ and $3.3 \mathrm{NBOs}$, i.e. the statistical values (see above). Interestingly, the strongest influence on 


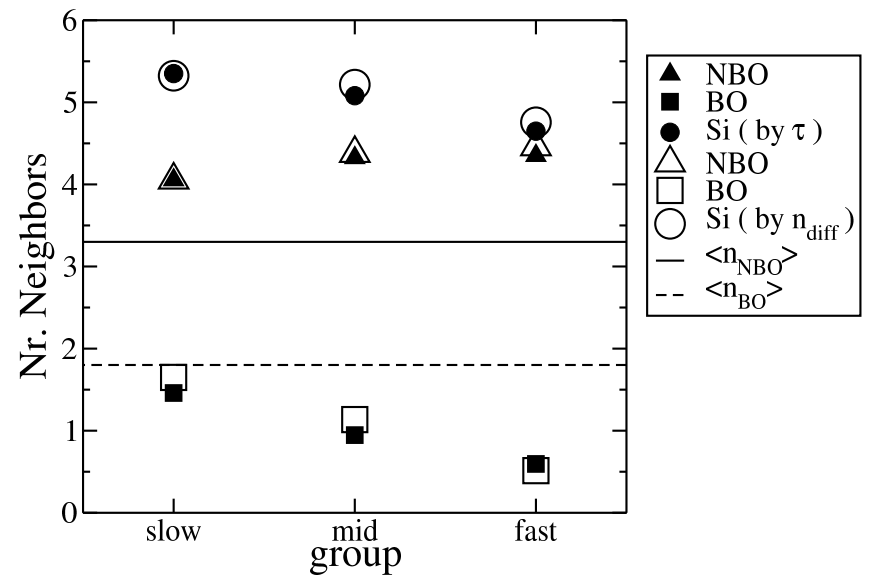

FIG. 5: Network species as next neighbors of sites

the mobility of a site is shown for the number of coordinated BOs, which decreases by $\approx 1$ from slow to fast sites. The number of silicon neighbors shows only a weak decrease in the same direction. For NBOs the correlation is reverse, but much weaker even on an absolute scale. The results are very similar when using $\tau$ or $n_{\text {diff }}$ to determine the mobility of the sites.

This result implies that in most cases a single BO in the nearest-neighbor shell is sufficient to immobilize ions on the sites around the BO. A possible underlying structure in the network that could determine the layout of conduction channels should therefore be related to the distribution of BOs. Similarly to the method described above, we sorted all sites into three groups according to the number of BOs in their 1st shell closer than $2.9 \AA$. Then the fraction of neighbors from each of these groups was counted. The results are given in Fig. 6] The dashed line again marks the statistical value, which is 0.33 in this case, corresponding to groups of one third of all sites. The expected trivial correlation among nearest neighbors is, of course, found. Most important, no correlation to the second nearest neighbor shell is found. We checked that also the NBOs do not display any correlations beyond the nearest neighbor shell. The lack of spatial correlations beyond the nearest neighbors strongly suggests that even the distribution of bridging oxygen atoms as the most relevant network property does not determine complete conduction channels in the system.

\section{DISCUSSION}

The presence of only one BO drastically slows down the ionic transport through nearby sites. An indication how this slowdown is effected can be obtained from the two examples shown in Fig[7. Here the local network structure is shown around one slow site (left) and one fast site. Cations are shown at the center as solid balls. The surrounding silicate network is shown as tubes,. The site as determined by us ${ }^{11}$ is represented by the translucent

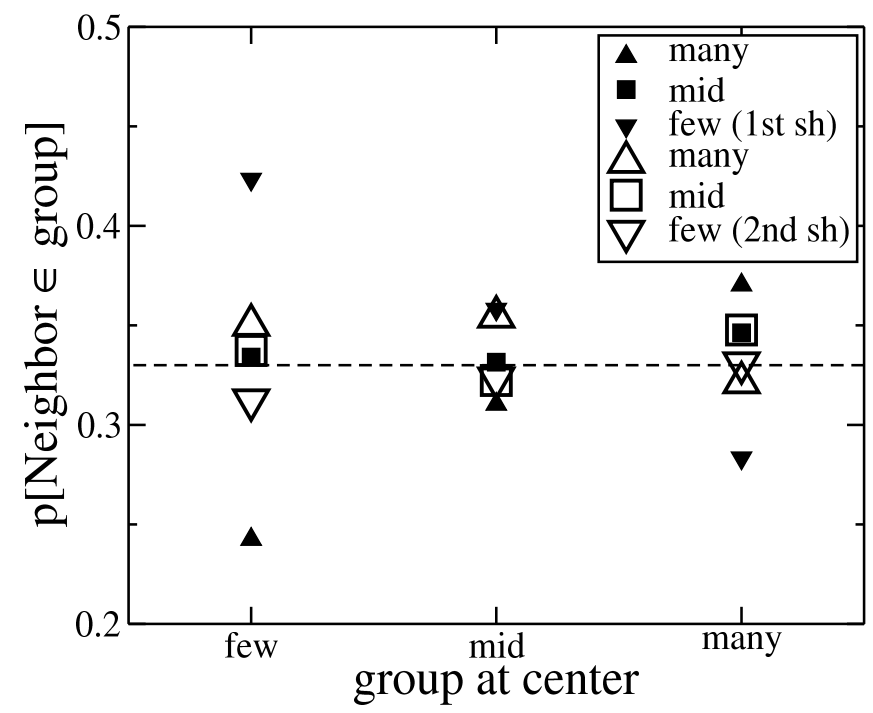

FIG. 6: Correlations in the number of neighboring BOs of sites

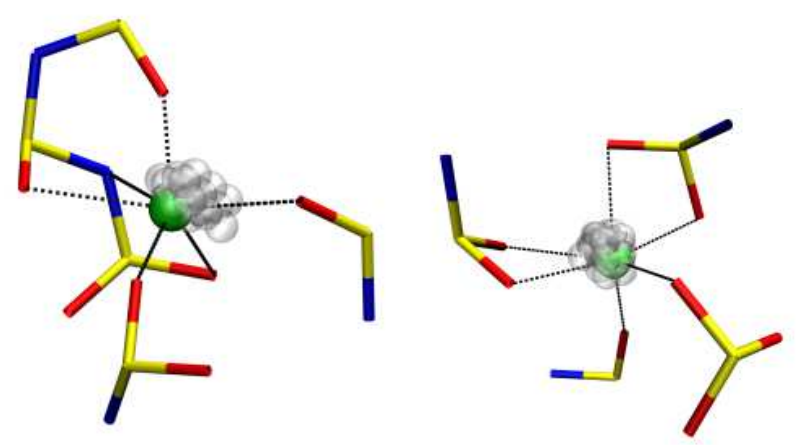

FIG. 7: Local structure around a slow site (left) and a fast site (created with the software vmd ${ }^{15}$ )

cloud surrounding the cation. Connections are drawn between the lithium ion and all oxygen atoms within the first neighbor shell. For the fast site, all six coordinating oxygen atoms are NBOs, which are bound to four different silicon atoms. For the slow site there is one BO among the neighbors. This $\mathrm{BO}$ is part of a silica chain segment that is also carrying three of the NBOs coordinating the cation. These NBOs are bound to three silicon atoms on different sides of the BO, with an additional BO inserted on one side in between. In total, a chain segment of 5 atoms is linked to the cation via four coordinating oxygen atoms. The chain is thus closely wrapped around a large part of the site. This situation suggests that BOs introduce high transport barriers for nearby sites because the silica chains joined by them block a large fraction of possible jump pathways.

The pathways through the system used for effective ionic transport follow continuous channels of sites visited by many ions. The passage of a large number of different ions through a specific site depends on two conditions. (i) 


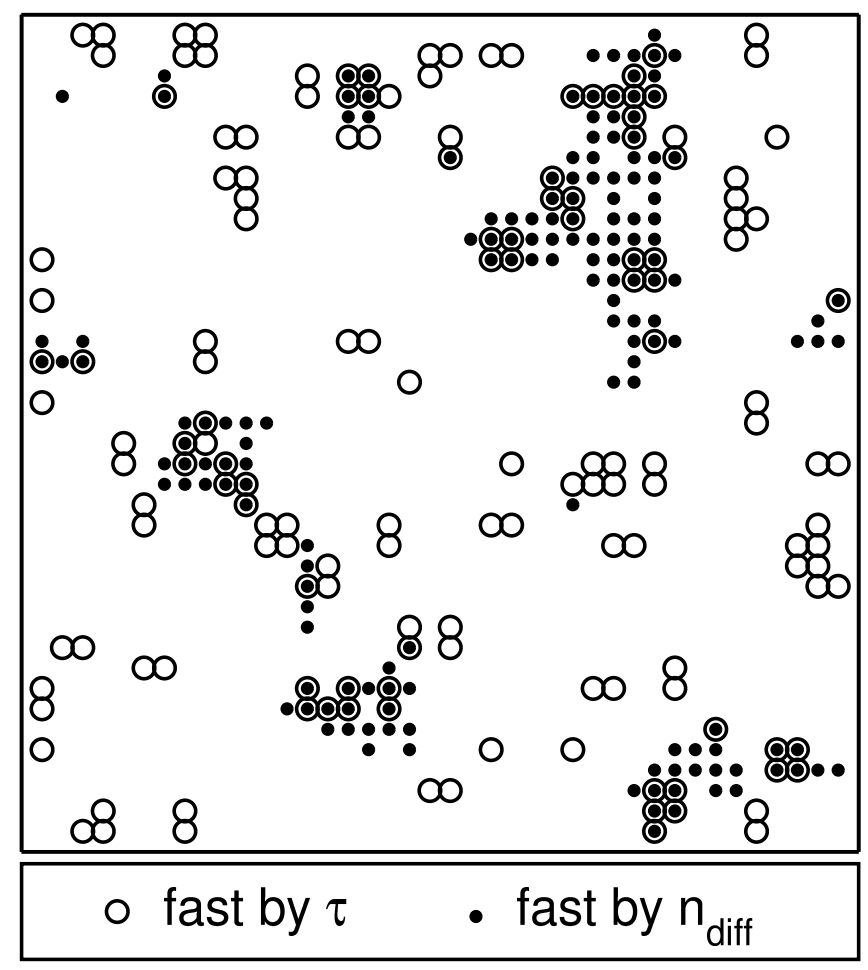

FIG. 8: Spatial correlations in a simple lattice model

A comparatively low average residence time of the sites is necessary but not sufficient to enable the passage of many ions. (ii) The presence of coordinating BOs strongly decreases the number of ions reaching a site, probably by introducing a local barrier. Both the distribution of sites with low $\tau$ and that of BOs in the structure show no long range order comparable to that found in the pathways traced by sites with high $n_{\text {diff }}$. They can therefore only impose constraints on the layout of these pathways, but not determine them. Therefore the emergence of conduction channels does not seem to require preformed channels provided by the network structure.

Now we show that on a qualitative level similar effects can be observed for the simple lattice model, introduced in Section I After performing long Monte Carlo simulations we determined the A- and B-sites similarly to our procedure for the silicate system. They are marked by filled dots and open circles, respectively. Interest- ingly, the three main observations from the silicate system are reproduced: (i) By definition the structure (here: the local energetic parameters) does not display correlations beyond the nearest neighbor shell. (ii) The B-sites are not spatially correlated beyond the nearest-neighbor shell. Actually, one clearly sees that sites with short residence time typically occur as pairs, because of the trivial correlations discussed above. (iii) The A-sites are spatially correlated, i.e. they are concentrated in a few larger blobs. These regions contain some of the groups of sites with low $\tau$, but also some sites with higher residence times. Correspondingly, some sites with low $\tau$ are not incorporated into regions with high $n_{\text {diff }}$. Sites visited by many different ions can of course not be completely isolated. The ions have to move away through other sites in the neighborhood to allow the following ions to enter. In our lattice model the regions best suited for this process are located by chance. The ions benefit from sites with very low residence times, they must reach them via other sites that are also not too slow, and they must avoid high barriers distributed through the lattice. Interestingly, the pathways are somewhat extended in both dimensions. This suggests that regions where many different particles may diffuse are extended in all directions. Actually, the channels, as obtained from the molecular dynamics simulations $\mathrm{E}^{\underline{\underline{ }}}$ show this property as well.

In summary, we have analyzed the nature of the conduction pathways in lithium silicate as well as a simple random lattice model. In both cases we do find spatial correlations in regions of high mobility. These structures do not seem to be supplemented by corresponding spatial correlations of network properties, following from the lack of long-range correlations of sites with BOs. Thus we would like to suggest that the formation of conduction channels is rather a statistical selection process in analogy to the formation of percolation paths in simple lattice models.

\section{Acknowledgments}

We thank M. Korth and H. Krieg for the help with the MC-simulations. Furthermore we acknowledge very helpful conversations with P. Jund. H. L. also acknowledges the support by the Fonds der Chemischen Industrie.
* hlammert@uni-muenster.de

† andheuer@uni-muenster.de

1 G. N. Greaves, J. Non-Cryst. Solids 71, 203 (1985).

2 A. T. W. Yap, H. Förster, and S. R. Elliott, Phys. Rev. Lett. 75, 3946 (1995).

3 B. Gee and H. Eckert, J. Phys. Chem 100, 3705 (1996).

4 A. Heuer, M. Kunow, M. Vogel, and R. D. Banhatti, Phys. Chem. Chem. Phys. 4, 3185 (2002).

5 J. Horbach, W. Kob, and K. Binder, Chem. Geol. 174, 87
(2001)

${ }^{6}$ P. Jund, W. Kob, and R. Jullien, Phys. Rev. B 64, 134303 (2002).

7 A. Meyer, H. Schober, and D. B. Dingwell, Europhys. Lett. 59, 708 (2002).

8 J. Horbach, W. Kob, and K. Binder, Phys. Rev. Lett. 88, 125502 (2002).

9 E. Sunyer, P. Jund, and R. Jullien, J. Phys.: Condens. Matter 15, S1659 (2003). 
10 J. C. Dyre and T. B. Schrøder, Rev. Mod. Phys. 72, 873 (2000).

11 H. Lammert, M. Kunow, and A. Heuer, Phys.Rev.Lett. 90, 215901 (2003).

12 N. Kamijo, K. Handa, and N. Umesaki, Mater. Trans, JIM 37, 927 (1996).

13 P. Florian, K. E. Vermillion, P. J. Grandinetti, I. Farnan, and J. F. Stebbins, J. Am. Chem. Soc. 118, 3493 (1996).

14 E. Sunyer, P. Jund, W. Kob, and R. Jullien, J. Non-Cryst. Solids 307, 939 (2003).

15 W. Humphrey, A. Dalke, and K. Schulten, J. Molec. Graphics 14, 33 (1996). 